\title{
Aplicação de métodos de otimização multiobjetivo para resolver o problema da dieta
}

\author{
Rúbia Mara de Oliveira Santos (D) Ronaldo Faria Garcia (D)
}

\section{Resumo}

Neste trabalho são investigados dois métodos empregados na resolução de problemas de otimização multiobjetivo, a saber, o método do Critério Global e o método $\epsilon$ Restrito. Ambos os métodos são aplicados ao problema da dieta com dois objetivos. As estratégias utilizadas são Aglutinação, onde os objetivos integram a função global, e Priorização, onde, um objetivo é privilegiado em relação ao outro.

Palavras-chave: Otimização Multiobjetivo; Método do Critério Global; Método $\epsilon$ Restrito.

\begin{abstract}
This work aims to present two methods for solving multiobjective optimization problems, the Global Criterion method and the $\epsilon$ Restricted method. The methods will be applied to the diet problem with two objectives. The strategies used are agglutination, where objectives integrate the global function, and prioritization, where one objective is privileged in relation to the other.
\end{abstract}

Keywords: Multiobjective Optimization; Global Criteria Method; $\epsilon$ Restricted method.

\section{Introdução}

A partir da década de 1970 tem sido crescente o emprego de modelos de otimização para representarem uma situação problema mais real, onde vários objetivos, em geral conflitantes, concorrem para a melhor solução de um problema, estabelecendo um problema de Otimização Multiobjetivo $[1,3]$. A Otimização Multiobjetivo distingue-se de todos os ramos da teoria de otimização quanto ao sentido que o conceito de solução do problema adquire. De fato, pode-se definir um problema de otimização multiobjetivo como otimização (nesse caso, minimização) de um vetor composto por funções escalares, escolhidas como forma de avaliar o impacto nas decisões factíveis do problema de acordo com diferentes índices de desempenho. Entretanto, sabe-se, funções vetoriais estão definidas em um conjunto parcialmente ordenado (por exemplo o $\mathbb{R}^{\mathrm{m}}, \mathrm{m} \geq 2$ ), e que portanto podem existir alternativas factíveis que não satisfaçam nenhuma relação de ordem do tipo $(\leq)$, inviabilizando dessa forma a utilização do conceito usual de solução ótima utilizado em problemas monobjetivos; mais informações encontram-se em $[1,3]$.

A solução do problema depende de como equilibrar as decisões para resolver os conflitos que surgem através das funções-objetivo. A dominância de Pareto é uma técnica que se adapta para resolver o problema. 
Um problema multiobjetivo genérico pode ser formulado no espaço das variáveis de decisão como

$$
\begin{array}{cc}
\mathrm{P}_{\mathrm{x}}: \operatorname{minimizar} & \mathrm{f}(\mathrm{x}) \\
\text { s.a. } & \mathrm{x} \in \mathscr{K},
\end{array}
$$

onde $\mathrm{f}=\left(\mathrm{f}_{1}, \ldots, \mathrm{f}_{\mathrm{m}}\right)$ e $\mathrm{m} \geq 2$. Observe que $\mathrm{f}: \mathrm{X} \subset \mathbb{R}^{\mathrm{n}} \rightarrow \mathbb{R}^{\mathrm{m}}$, isto é, cada ponto $\mathrm{x} \in \mathrm{X}$ é mapeado no espaço real m-dimensional.

A utilização de problemas monobjetivos para descrever e gerar as soluções eficientes estão associadas às condições de otimalidade dos problemas escalares [3]. A seguir tem-se um método de aglutinação para a resolução de problemas multiobjetivos. Uma solução ótima é um vetor de variáveis de decisão, o qual sempre minimiza a função global $\mathscr{F}(\cdot)$, respeitando as restrições do problema. A função global $\mathscr{F}(\mathrm{x})$ do problema $\mathrm{P}_{\mathrm{x}}$ é obtida por:

$$
\mathscr{F}(\mathrm{x})=\frac{\mathrm{f}_{1}^{*}(\mathrm{x})-\mathrm{f}_{1}(\mathrm{x})}{\mathrm{f}_{1}^{*}(\mathrm{x})}+\frac{\mathrm{f}_{2}^{*}(\mathrm{x})-\mathrm{f}_{2}(\mathrm{x})}{\mathrm{f}_{2}^{*}(\mathrm{x})}+\ldots+\frac{\mathrm{f}_{\mathrm{m}}^{*}(\mathrm{x})-\mathrm{f}_{\mathrm{m}}(\mathrm{x})}{\mathrm{f}_{\mathrm{m}}^{*}(\mathrm{x})}
$$

$\mathrm{f}_{1}^{*}(\mathrm{x}), \mathrm{f}_{2}^{*}(\mathrm{x}), \ldots, \mathrm{f}_{\mathrm{m}}^{*}(\mathrm{x})$ são soluções ótimas individuais das funções-objetivo $\mathrm{f}_{1}(\mathrm{x}), \mathrm{f}_{2}(\mathrm{x}),, \ldots, \mathrm{f}_{\mathrm{m}}(\mathrm{x})$, respectivamente. Dessa forma a solução eficiente do problema $\mathrm{P}(\mathrm{x})$ resume-se em encontrar a solução ótima-Pareto do problema monobjetivo.

$$
\begin{array}{cr}
\mathscr{P} \text { : minimizar } & \mathscr{F}(\mathrm{x}) \\
\text { s.a. } & \mathrm{x} \in \mathscr{K} .
\end{array}
$$

O problema $\mathscr{P}$ é um problema com estratégia de aglutinação $[1,3]$ e denominado Método do Critério Global.

Teorema 1. Se $\mathrm{x}^{*} \in \operatorname{efi}(\mathscr{C})$, então existe um inteiro $\mathrm{k} \in \mathscr{T}=\{1,2, \ldots, \mathrm{m}\}$ e números reais $\epsilon_{\mathrm{j}}, \mathrm{j}=$ $1,2 \ldots, \mathrm{m} \quad(\mathrm{j} \neq \mathrm{k})$ tais que $\mathrm{x}^{*}$ resolve

$$
\begin{array}{cl}
\mathrm{P}_{\mathrm{k}}(\epsilon): & \operatorname{minimizar}_{\mathrm{x} \in \mathscr{C}} \quad \mathrm{f}_{\mathrm{k}}(\mathrm{x}) \\
\text { s.a } & \mathrm{f}_{\mathrm{j}}(\mathrm{x}) \leq \epsilon_{\mathrm{j}}, \quad 8 \mathrm{j} \neq \mathrm{k},
\end{array}
$$

onde $\epsilon$ está definido em $\varepsilon_{\mathrm{k}}=\left\{\epsilon=\left(\epsilon_{1} \ldots, \epsilon_{\mathrm{k}-1}, \epsilon_{\mathrm{k}+1}, \ldots, \epsilon_{\mathrm{m}}\right): \mathscr{C}_{\mathrm{k}}(\epsilon) \neq \varnothing\right\}$ e

$$
\mathscr{C}_{\mathrm{k}}(\epsilon)=\left\{\mathrm{x} \in \mathscr{C}: \mathrm{f}_{\mathrm{j}}(\mathrm{x}) \leq \epsilon_{\mathrm{j}} 8 \mathrm{j} \neq \mathrm{k}\right\} .
$$

O problema $\mathrm{P}_{\mathrm{k}}(\epsilon)$ é um problema de priorização $[1,3]$ denominado de Método $\epsilon$ Restrito. O método exato $\epsilon$-restrito consiste em priorizar na otimização o objetivo mais importante, respeitando as restrições impostas pelo problema.

O princípio utilizado neste trabalho é a noção de Equilíbrio Cooperativo de Pareto aplicado em problemas envolvendo duas funções-objetivo, ou seja, um problema de otimização biobjetivo [2]. $\mathrm{Na}$ Figura 1 tem-se uma função biobjetivo que associa vetores decisão $\left(\mathrm{x}_{1}, \mathrm{x}_{2}\right)$ do conjunto factível $\mathscr{6}$ ao conjunto de espaço de objetivos $\mathscr{H}$. 


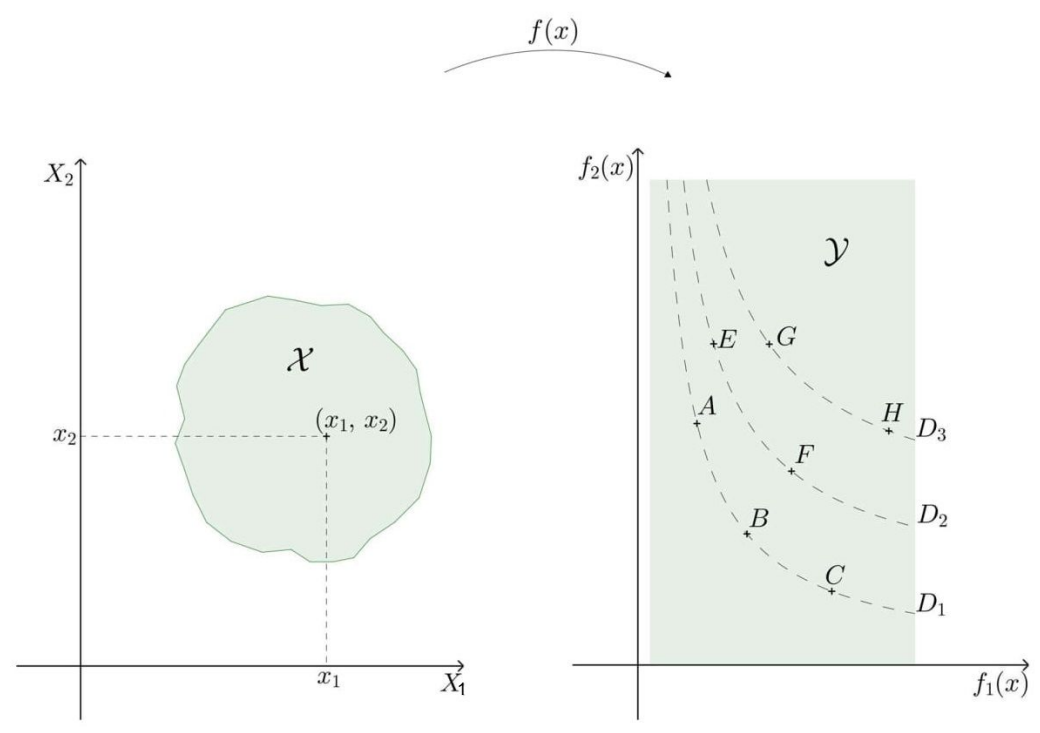

Figura 1: Conjunto de Soluções Factíveis; Espaço Objetivo Factível e Linhas de Dominância.

Através das linhas de dominâncias $\mathrm{D}_{1}, \mathrm{D}_{2}$ e $\mathrm{D}_{3}$ as soluções eficientes $\mathrm{A}, \mathrm{B}$ e C não são dominadas por nenhuma solução eficiente, pois pertencem à fronteira de Pareto; a solução A domina a solução F, que, por sua vez, domina a solução G.

\section{Aplicação: Problema da Dieta.}

Como propiciar uma refeição saudável consumindo o mínimo de calorias obtendo o menor custo possível? O problema consiste em minimizar dois objetivos, estabelecendo um problema de otimização biobjetivo [2].

Dados divulgados pela Organização Mundial da Saúde (OMS) mostram que a diabetes é uma doença que atinge uma em cada 11 pessoas no mundo, passando de 108 milhões em 1980 para 422 milhões em 2014. A projeção feita pela Internatinal Diabetes Federation (IDF) é de que em 2030 haja um aumento de $123 \%$ dos casos. No Brasil, segundo o ministério da Saúde, entre os anos 2006 e 2016 os casos de diabetes cresceram $6,8 \%$, e o número de pessoas com a doença é aproximadamente de $8,9 \%$ da população brasileira. A previsão da IDF é de que 23 milhões de pessoas tenham a doença no Brasil em 2045; veja [2, 4]. A diabete é uma doença crônica que exige uma alimentação mais saudável, entre os quais a maçã e a batata-doce são alimentos imprescindíveis no preparo de uma refeição, pois a batata-doce é um aliado de quem pratica esportes e possui um baixo índice glicêmico - isso quer dizer que seus carboidratos são absorvidos lentamente pelo organismo, o que ajuda no controle da doença. Por outro lado, a maçã é uma fruta que possui na sua casca uma fibra chamada pectina, importante para o controle da glicemia e para reduzir o mau colesterol e também como a batata-doce, a maçã tem baixo índice glicêmico.

Pretende-se preparar uma refeição saudável, ou seja, com um número mínimo de calorias, porém com menor custo possível. A refeição tem que conter maça e/ou batata-doce, sendo que a quantidade máxima de maçã e batata-doce é de 200 g; a quantidade mínima de carboidratos ingerida 
pelo consumo da batata-doce por refeição é de 20 g; e a quantidade mínima de proteínas é de 1,0 g.

O custo, a quantidade de calorias, quantidade de carboidratos e proteínas são fornecidos pela Tabela 1 .

\begin{tabular}{ccccc}
\hline Por $100 \mathrm{~g}$ & Custo(centavos) & Calorias & Carboidrato & Proteínas \\
\hline Maçã & 50 & $52 \mathrm{Kcal}$ & $10 \mathrm{~g}$ & $0,3 \mathrm{~g}$ \\
\hline Batata-Doce & 20 & $86 \mathrm{Kcal}$ & $20 \mathrm{~g}$ & $1,5 \mathrm{~g}$ \\
\hline
\end{tabular}

Tabela 1: Tabela de Alimentos

O problema consiste em encontrar uma solução que minimize o número de calorias e o custo, sendo modelado como um problema biobjetivo. Dessa forma segue o problema $\mathrm{P}$.

$$
\begin{array}{cc}
\text { P : minimizar } & \mathrm{f}_{1}\left(\mathrm{x}_{1}, \mathrm{x}_{2}\right)=50 \mathrm{x}_{1}+20 \mathrm{x}_{2} \\
& \mathrm{f}_{2}\left(\mathrm{x}_{1}, \mathrm{x}_{2}\right)=52 \mathrm{x}_{1}+86 \mathrm{x}_{2} \\
\text { s.a. } & \mathrm{x}_{1}+\mathrm{x}_{2} \leq 2 \\
& 10 \mathrm{x}_{1}+20 \mathrm{x}_{2} \geq 20 \\
& 0,3 \mathrm{x}_{1}+1,5 \mathrm{x}_{2} \geq 1 \\
& \mathrm{x}_{1}, \mathrm{x}_{2} \geq 0
\end{array}
$$

com $\mathrm{x}_{1}$ sendo a quantidade de maçã e $\mathrm{x}_{2}$ a quantidade de batata-doce, em centenas de gramas.

Inicialmente será determinado o espaço factível, o espaço de objetivos e em seguida aplicado o Método do Critério Global e o Método $\epsilon$ Restrito. O espaço factível é representado pela Figura 2.

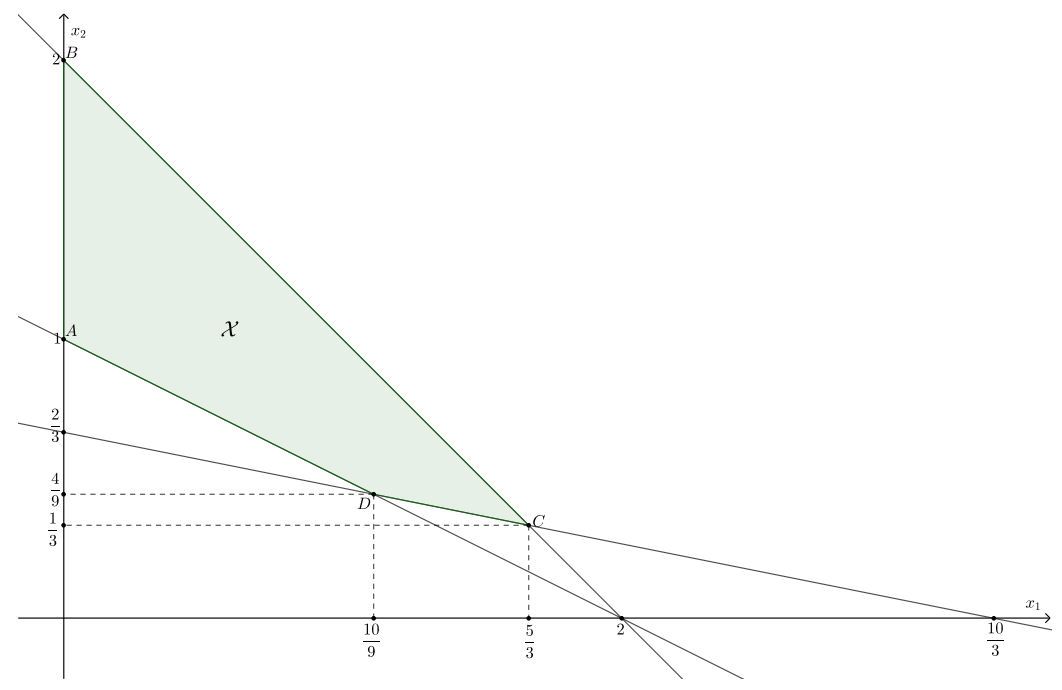

Figura 2: Espaço Factível $\mathscr{6}$ 
Observe que $\mathscr{C}$ é um polígono com vértices $\mathrm{A}(0,1), \mathrm{B}(0,2), \mathrm{C}\left(\frac{5}{3}, \frac{1}{3}\right)$ e $\mathrm{D}\left(\frac{10}{9}, \frac{4}{9}\right)$. Encontrando o valor numérico das funções $\mathrm{f}_{1}\left(\mathrm{x}_{1}, \mathrm{x}_{2}\right)$ e $\mathrm{f}_{2}\left(\mathrm{x}_{1}, \mathrm{x}_{2}\right)$ nos vértices desse polígono, tem-se $\mathrm{f}_{1}(0,1)=20$; $\mathrm{f}_{1}(0,2)=40 ; \mathrm{f}_{1}\left(\frac{5}{3}, \frac{1}{3}\right)=90$ e $\mathrm{f}_{1}\left(\frac{10}{9}, \frac{4}{9}\right)=\approx 64,44$. Por outro lado, $\mathrm{f}_{2}(0,1)=86 ; \mathrm{f}_{2}(0,2)=172 ;$

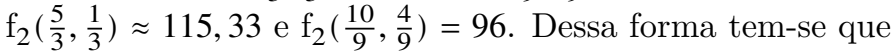

$$
f_{1}^{*}\left(x_{1}, x_{2}\right)=20 \text { e } f_{2}^{*}\left(x_{1}, x_{2}\right)=86
$$

A Figura 3 representa o espaço de objetivos do problema.

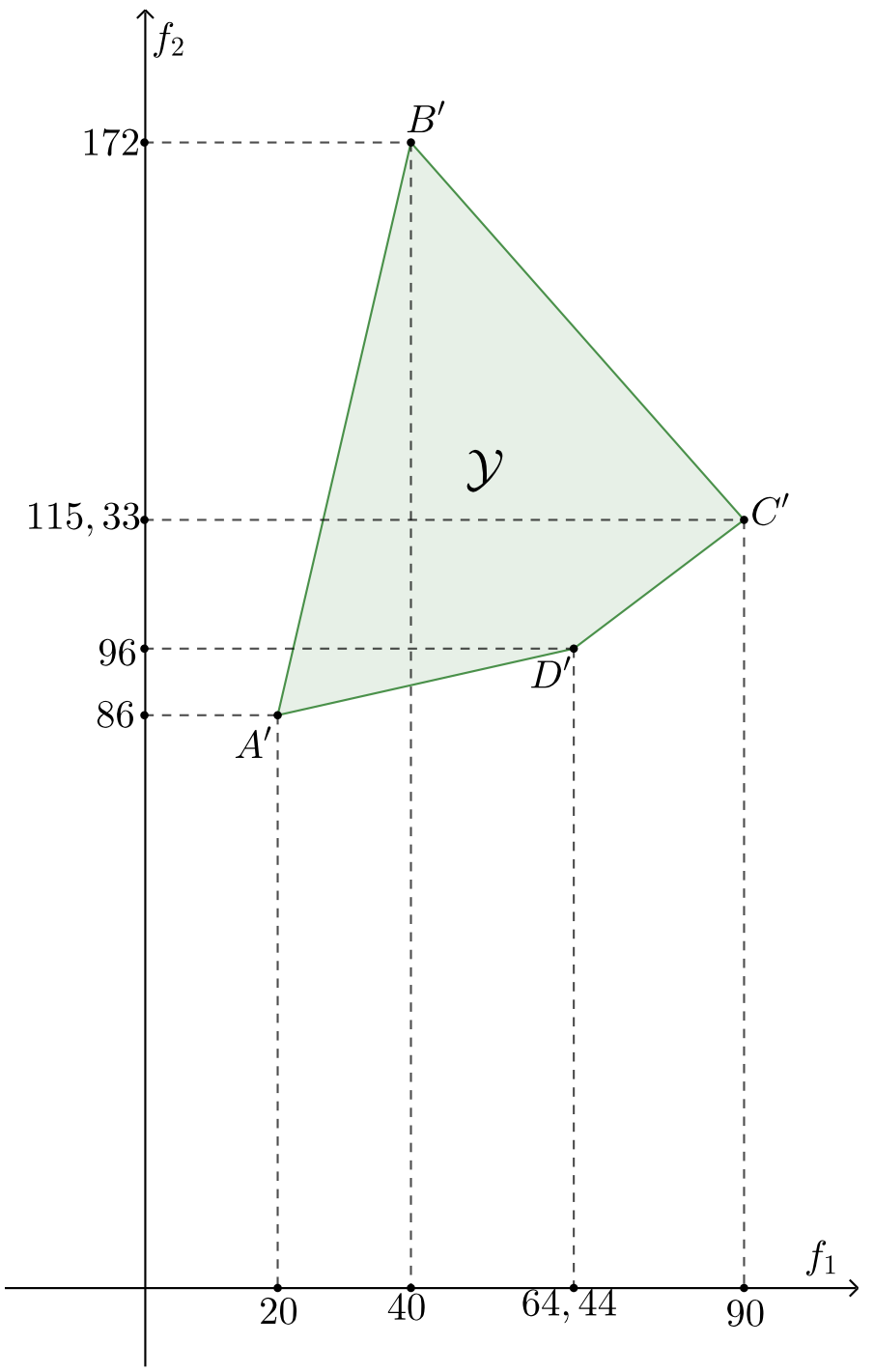

Figura 3: Espaço de Objetivos 
Pelo método do critério global, a função global $\mathscr{F}\left(\mathrm{x}_{1}, \mathrm{x}_{2}\right)$ é dada por:

$$
\begin{gathered}
\mathscr{F}\left(\mathrm{x}_{1}, \mathrm{x}_{2}\right)=\frac{\mathrm{f}_{1}^{*}\left(\mathrm{x}_{1}, \mathrm{x}_{2}\right)-\mathrm{f}_{1}\left(\mathrm{x}_{1}, \mathrm{x}_{2}\right)}{\mathrm{f}_{1}^{*}\left(\mathrm{x}_{1}, \mathrm{x}_{2}\right)}+\frac{\mathrm{f}_{2}^{*}\left(\mathrm{x}_{1}, \mathrm{x}_{2}\right)-\mathrm{f}_{2}\left(\mathrm{x}_{1}, \mathrm{x}_{2}\right)}{\mathrm{f}_{2}^{*}\left(\mathrm{x}_{1}, \mathrm{x}_{2}\right)} \\
\mathscr{F}\left(\mathrm{x}_{1}, \mathrm{x}_{2}\right)=\frac{-267 \mathrm{x}_{1}}{86}-2 \mathrm{x}_{2}+2
\end{gathered}
$$

Minimizando $\mathscr{F}\left(\mathrm{x}_{1}, \mathrm{x}_{2}\right)$ e Reescrevendo na forma padrão tem-se:

$$
\begin{array}{rc}
\operatorname{minimizar} & \mathscr{F}\left(\mathrm{x}_{1}, \mathrm{x}_{2}\right)=\frac{-267 \mathrm{x}_{1}}{86}-2 \mathrm{x}_{2}+2 \\
\text { s.a. } & \mathrm{x}_{1}+\mathrm{x}_{2}+\mathrm{x}_{3}=2 \\
& \mathrm{x}_{1}+2 \mathrm{x}_{2}-\mathrm{x}_{4}=2 \\
& 3 \mathrm{x}_{1}+15 \mathrm{x}_{2}-\mathrm{x}_{5}=10
\end{array}
$$

As restrições formam um sistema linear com 3 equações e 5 variáveis, onde o número de variáveis não básicas é igual a 5-3=2, e o total de soluções básicas é $\mathrm{C}_{5,3}=10$.

\begin{tabular}{cccccccc}
\hline Souções Básicas & $\mathrm{x}_{1}$ & $\mathrm{x}_{2}$ & $\mathrm{x}_{3}$ & $\mathrm{x}_{4}$ & $\mathrm{x}_{5}$ & $\mathrm{x}^{*}$ & $\mathrm{z}$ \\
\hline 1 & 0 & 0 & 2 & -2 & -10 & $(0,0)$ & $\mathrm{z}_{1}=69$ \\
\hline 2 & 0 & 2 & 0 & 2 & 20 & $(0,2)$ & $\mathrm{z}_{2}=-2$ \\
\hline 3 & 0 & 1 & 1 & 0 & 5 & $(0,1)$ & $\mathrm{z}_{3}=0$ \\
\hline 4 & 0 & $-\frac{2}{3}$ & $\frac{8}{3}$ & $-\frac{10}{3}$ & 0 & $\left(0, \frac{2}{3}\right)$ & $\mathrm{z}_{4}=69$ \\
\hline 5 & 2 & 0 & 0 & 0 & 4 & $(2,0)$ & $\mathrm{z}_{5}=69$ \\
\hline 6 & 2 & 0 & 0 & 0 & -4 & $(2,0)$ & $\mathrm{z}_{6}=69$ \\
\hline 7 & $\frac{10}{3}$ & 0 & $-\frac{4}{3}$ & $\frac{4}{3}$ & 0 & $\left(\frac{10}{3}, 0\right)$ & Não Factível \\
\hline 8 & - & - & 0 & 0 & - & - & Não Factível \\
\hline 9 & $\frac{5}{3}$ & $\frac{1}{3}$ & 0 & $\frac{1}{3}$ & 0 & $\left(\frac{5}{3}, \frac{1}{3}\right)$ & $\mathrm{z}_{9} \approx-3,836$ \\
\hline 10 & $\frac{10}{9}$ & $\frac{4}{9}$ & $\frac{4}{9}$ & 0 & 0 & $\left(\frac{10}{9}, \frac{4}{9}\right)$ & $\mathrm{z}_{10} \approx-2,388$ \\
\hline
\end{tabular}

Tabela 2: Soluções Básicas

Na aplicação do método do Critério Global tem-se a minimização da função global; dessa forma a solução ótima do problema é a solução básica 9 onde, $\mathrm{x}^{*}=\left(\frac{5}{3}, \frac{1}{3}\right) ; \mathrm{z}_{9} \approx-3,836$, e $\mathrm{f}_{1}\left(\frac{5}{3}, \frac{1}{3}\right)=90 \mathrm{e}$ $\mathrm{f}_{2}\left(\frac{5}{3}, \frac{1}{3}\right)=115,33$. Logo, o preço da refeição é de 90 centavos a cada $100 \mathrm{~g}$ e a quantidade ótima de calorias é de 115,33 Kcal.

Considerando as mesmas restrições e supondo que o custo para cada $100 \mathrm{~g}$ de alimentos seja menor ou igual a 80 centavos, dessa forma o problema de otimização a resolver é:

$$
\begin{aligned}
\mathrm{P}(\epsilon) \text { : minimizar } & \mathrm{f}_{2}\left(\mathrm{x}_{1}, \mathrm{x}_{2}\right)=52 \mathrm{x}_{1}+86 \mathrm{x}_{2} \\
& \mathrm{f}_{1}\left(\mathrm{x}_{1}, \mathrm{x}_{2}\right)=50 \mathrm{x}_{1}+20 \mathrm{x}_{2} \leq \epsilon_{1}=80 \\
\text { s.a. } \quad & \mathrm{x}_{1}+\mathrm{x}_{2} \leq 2 \\
& \mathrm{x}_{1}+2 \mathrm{x}_{2} \geq 2
\end{aligned}
$$




$$
3 \mathrm{x}_{1}+15 \mathrm{x}_{2} \geq 10
$$

A estratégia utilizada para a resolução do problema $\mathrm{P}(\epsilon)$ é a priorização, pois a função $\mathrm{f}_{1}\left(\mathrm{x}_{1}, \mathrm{x}_{2}\right)$ está sendo priorizada quando se considera o limite de 80 centavos para cada $100 \mathrm{~g}$ de alimento. O método para resolver o problema será $\epsilon$ Restrito.

O espaço factível do problema $\mathrm{P}(\epsilon)$ é representado pela Figura 4.

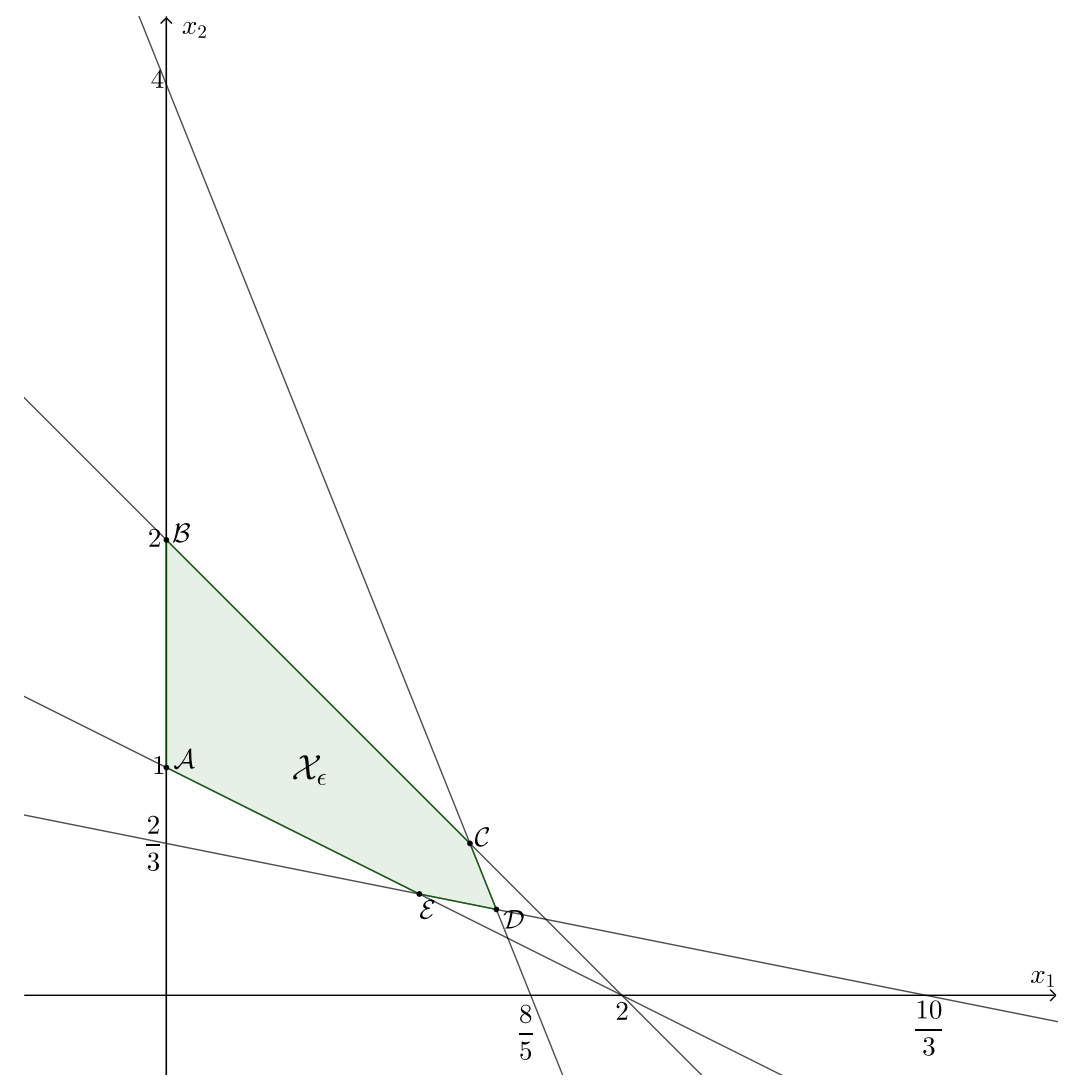

Figura 4: Espaço Factível $\mathscr{C}_{\epsilon}$

Observe que $\mathscr{C}_{\epsilon}$ é um polígono com vértices $\mathscr{A}(0,1), \mathscr{B}(0,2), \mathscr{C}\left(\frac{4}{3}, \frac{2}{3}\right), \mathscr{D}(1,45 ; 0,38)$ e $\mathscr{E}\left(\frac{10}{9}, \frac{4}{9}\right)$, e, encontrando o valor numérico da função $\mathrm{f}_{2}(\mathrm{x})=52 \mathrm{x}_{1}+86 \mathrm{x}_{2}$ em cada um desses pontos, tem-se: $\mathrm{f}_{2}(0,1)=86 ; \mathrm{f}_{2}(0,2)=172 ; \mathrm{f}_{2}\left(\frac{4}{3}, \frac{2}{3}\right) \approx 126,66 ; \mathrm{f}_{2}(1,45 ; 0,38) \approx 108,8$ e $\mathrm{f}_{2}\left(\frac{10}{9}, \frac{4}{9}\right)=96$.

A solução ótima para o problema através do método $\epsilon$ Restrito é $x^{*}=(0,1)$ e $f_{2}(0,1)=86$, ou seja, supondo $\epsilon_{1}=80$ o consumo de maçã não ocorrerá e haverá o consumo de uma batata-doce com 86 kcal. Caso seja necessário o consumo dos dois alimentos, a solução ótima para o problema é $\mathrm{x}^{*}=\left(\frac{10}{9}, \frac{4}{9}\right)$ e tem-se 96 kcal consumidas.

A solução obtida pelo método $\epsilon$-restrito está relacionada com a escolha de $\epsilon$ e da priorização da função objetivo, o que resulta em um controle de cobertura da fronteira eficiente, obtendo melhor resultado que o método do critério global.Ambos os métodos,porém, apresentaram simplicidade de resolução. 


\section{Comentários finais}

Este trabalho apresentou a aplicação de um problema biobjetivo que estabelece uma refeição saudável para diabéticos, na qual foram utilizados dois métodos para a resolução do problema, o método do Critério Global e o método $\epsilon$ Restrito, com estratégias de aglutinação e priorização, respectivamente. Apesar da dificuldade da escolha do $\epsilon$ na aplicação do método $\epsilon$ Restrito, foi verificado melhor resultado quando comparado com o método do Critério Global, pois caracterizou uma cobertura maior da fronteira eficiente. Ambos os métodos apresentaram simplicidade na aplicação. Em trabalhos futuros espera-se ampliar o problema da dieta para mais de dois critérios e aplicar os métodos nos problemas propostos em [4].

\section{Agradecimentos}

Os autores agradecem à Universidade Federal de Mato Grosso do Sul pelo suporte à pesquisa deste trabalho.

\section{Referências}

[1] Ferreira, P. A. V. Otimização multiobjetivo: teoria e aplicações. Tese (Livre Docência em Engenharia Elétrica) Universidade Estadual de Campinas, Campinas, 1999.

[2] Garcia, R. F. Funções convexas no $\mathbb{R}^{2}$ e introdução à otimização biobjetivo. Dissertação (Mestrado Profissional em Matemática em Rede Nacional), Universidade Federal de Mato Grosso do Sul, Campo Grande, 2019.

[3] Oliveira, R. M. Algoritmos de busca global para problemas de otimização geométricos e multiplicativos. Tese (Doutorado em Engenharia Elétrica). Universidade Estadual de Campinas, Campinas, 2005.

[4] Amin, S. H., Gow, S. M. and Zhang, G. Selection of food items for diet problem using a multi-objective approach under uncertainty. Application of Decision Science in Business and Management. IntechOpen, 181. 2019.

Rúbia Mara de Oliveira Santos Instituto de Matemática

Universidade Federal de Mato Grosso do Sul <rubiaoliveira@gmail.com>

Ronaldo Faria Garcia Mestrado Profissional em Matemática em Rede Nacional <ronaldofgarcia@yahoo.com.br>

Recebido: 06/10/2020

Publicado: 26/03/2021 